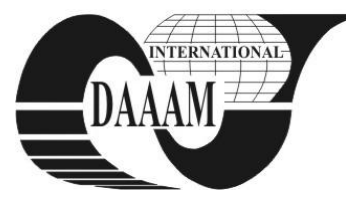

\title{
DYNAMIC ANALYSIS OF THE OIL ROD PUMPING SYSTEM MECHANISM
}

\section{DUMITRU, N[icolae]; BAILA, A[drian]; CRACIUNOIU, N[icolae] \& MALCIU, R[aluca]}

\begin{abstract}
The paper proposes a dynamic analysis of an artificial pumping system mechanism used for oil deep extraction. This dynamic analysis involves the determination of the kinematic and dynamic parameters characterizing the vibrations of the unit pumping mechanism elements. The variation law of the mechanism generalized coordinate is experimentally determined. Kinematic models were developed for the links longitudinal and transverse vibrations, which were processed by computer and led to time variation laws of longitudinal and transverse vibration displacements, respectively, speeds and accelerations defining the system vibratory motion. These laws were verified and validated by experimental research

Key words: rod pumping system, mathematical model, linearelastic displacements, vibrations
\end{abstract}

\section{INTRODUCTION}

Artificial pumping systems for deep extraction have a significant share in most countries with developed oil industry, both as wells number, and as flow rate, because of its advantages in terms of design simplicity and ease of servicing.

Pumping systems existing design mainly uses rough formulas and procedures based on analytical approaches of conventional oil / gas fields.

A calculation method for the studied rod pumping system design showed in Fig. 1 was presented in (Liu et al., 2011), where the mathematical model was determined based on the kinematic and dynamic analysis of the pumping system.

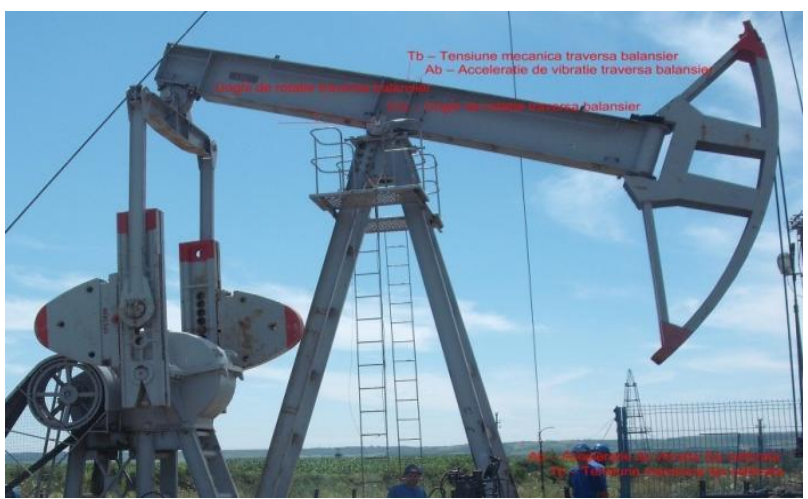

Fig. 1. Rod pumping system

Polished rod loads calculation usually took into account only static and inertial loads, and driving torque calculation used, in general, empirical formulas and correction factors. Polished rod loads calculation approaches in the literature include formulas of American Petroleum Institute (API), of Vilnovsky, Vilnovsky-Adowning, Emory Kemler and Mills.

These relationships modify and apply a variety of simplifying assumptions and analytical approaches to calculate the loads limits. They also considered only static and inertial loads, neglecting the vibrations and friction loads.

\section{THEORETICAL CONSIDERATIONS}

In order to achieve the dynamic analysis, the pumping unit mechanism was studied in the hypothesis that, in terms of structural elements, it consists of a driving link and two dyads: the RRR dyad, presented in Fig.2, and the RRT dyad presented in Fig. 3.

The mathematical model of longitudinal and transverse vibrations of the RRR dyad shown in Fig. 2 was developed and solved considering the links linear elastic straight bars.

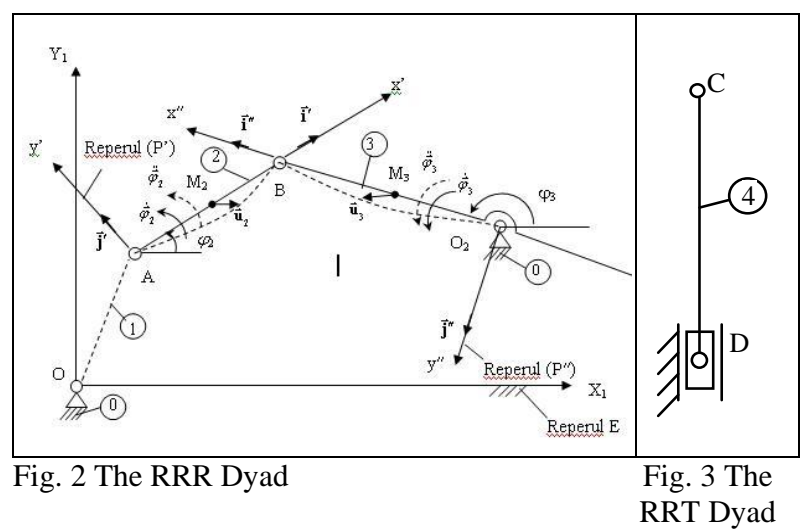

The vibrations mathematical model of a linear-elastic rectilinear kinematic element, with constant section, in a plane moving was deduced in (Buculei, 1981), having the form of a decoupled equations system.

By customizing it for the link 2 of the RRR dyad, the following mathematical model was obtained:

$$
\begin{aligned}
& c^{2} \frac{\partial^{2} u_{2}^{\prime}}{\partial x^{2}}-\frac{\partial^{2} u_{2}^{\prime}}{\partial t^{2}}+\dot{\varphi}_{2}^{2} u_{2}^{\prime}+\left(\dot{y}_{A} \dot{\varphi}_{2}-\ddot{x}_{A}+\dot{\varphi}_{2}^{2} x\right)=0 ; \\
& E I_{2} \frac{\partial^{4} u_{2}^{\prime \prime}}{\partial x^{4}}+\rho A_{2} \frac{\partial^{2} u_{2}^{\prime \prime}}{\partial t^{2}}+\dot{\varphi}_{2}^{2} A_{2} \rho u_{2}^{\prime \prime}+ \\
& +\left(\rho A_{2} \ddot{y}_{A}+\rho A_{2} \dot{\varphi}_{2} \dot{x}_{A}+\rho A_{2} \ddot{\varphi}_{2} x\right)=0
\end{aligned}
$$

where: $c^{2}=\frac{E}{\rho} ; \mathrm{A}_{2}$ - cross-sectional area of the dyad element; E - Young modulus; $\rho$ - element linear specific mass; $\mathrm{I}_{2}$ - the cross-sectional geometric moment of inertia of the dyad element relative to its neutral axis; $\vec{u}_{2}(x, t)=u_{2}^{\prime}(x, t) \vec{i}^{\prime}+u_{2}^{\prime \prime}(x, t) \vec{j}^{\prime}$ - linear elastic displacement of the dyad element (2); $u_{2}^{\prime}(x, t)$-longitudinal displacement;

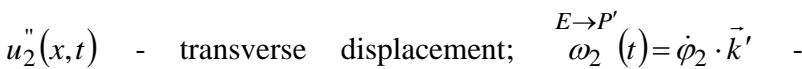
instantaneous absolute angular velocity of $\left(\mathrm{P}^{\prime}\right)$ marker with respect to (E), the exterior marker; $\begin{gathered}E \rightarrow P^{\prime} \\ \varepsilon_{2}\end{gathered}(t)=\ddot{\varphi}_{2} \cdot \vec{k}^{\prime}$ instantaneous absolute angular acceleration of $\left(\mathrm{P}^{\prime}\right)$ marker with respect to (E), the exterior marker; $\vec{v}_{A}^{E}(t)=\dot{x}_{A}(t) \vec{i}^{\prime}+\dot{y}_{A}(t) \vec{j}^{\prime}$ - 
the A pair velocity with respect to $(\mathrm{E})$, the exterior marker; $\vec{a}_{A}^{E}(t)=\ddot{x}_{A}(t) \vec{i}^{\prime}+\ddot{y}_{A}(t) \vec{j}^{\prime}-$ the A pair acceleration with respect to $(\mathrm{E})$, the exterior marker.

The kinematic parameters used in the mathematical model (1) are known from a prior kinematic analysis.

Using Laplace and Fourier integral transformations and taking into account the boundary conditions, the initial conditions and the known data for the RRR dyad as rigid model, we obtained the solutions of the mathematical model (1), representing the linear-elastic deformations components of the dyad links during the temporary motion subrange $\left[\mathrm{t}_{\mathrm{k}}, \mathrm{t}_{\mathrm{k}+1}\right], \mathrm{k}$ $=\overline{0, n}$. These solutions have the following form for the link (2):

$$
\begin{aligned}
& u_{2 k}^{\prime}(x, t)=\frac{2}{L_{2}} \sum_{n=1}^{\infty}\left\{\frac { 2 } { c ^ { 2 } \alpha _ { n } ^ { 2 } - \dot { \varphi } _ { 2 k } ^ { 2 } } \left[\frac{L_{2}}{\pi \cdot n}\left(1+(-1)^{n+1}\right) .\right.\right. \\
& \left.\cdot\left(\dot{y}_{A k} \dot{\varphi}_{2 k}-\ddot{x}_{A k}\right)+\frac{L_{2}^{2}}{\pi \cdot n}(-1)^{n+1} \dot{\varphi}_{2 k}^{2}\right] . \\
& \left.\cdot \sin ^{2}\left(\frac{\sqrt{c^{2} \alpha_{n}^{2}-\dot{\varphi}_{2 k}^{2}}}{2} \cdot t\right)\right\} \sin \left(\alpha_{n} x\right) ; \\
& u_{2 k}^{\prime \prime}(x, t)=\frac{2}{\rho A_{2} L_{2}} \sum_{n=1}^{\infty}\left\{-\frac{2 \rho A_{2}}{E I_{2} \alpha_{n}^{4}+\rho A_{2} \dot{\varphi}_{2 k}^{2}} \cdot\right. \\
& \cdot\left[\rho A_{2} \ddot{y}_{A k} \frac{L_{2}}{\pi \cdot n}\left(1+(-1)^{n+1}\right)+\right. \\
& \left.+\rho A_{2} \dot{x}_{A k} \dot{\varphi}_{2 k} \frac{L_{2}}{\pi \cdot n}\left(1+(-1)^{n+1}\right)+\rho A_{2} \ddot{\varphi}_{2 k}(-1)^{n+1} \frac{L_{2}^{2}}{\pi \cdot n}\right] \\
& \left.\cdot \sin ^{2}\left(\sqrt{\frac{E I_{2} \alpha_{n}^{4}+\rho A_{2} \dot{\varphi}_{2 k}^{2}}{\rho A_{2}}} \cdot t\right)\right\} \sin \left(\alpha_{n} x\right)
\end{aligned}
$$

where: $\alpha_{\mathrm{n}}=\frac{\pi \cdot n}{L_{2}} ; L_{2}$ - the length of the link (2) in undistorted state. $\dot{\varphi}_{2 k}=\omega_{2 k}$ - instantaneous absolute angular velocities of the dyad link (2); $\ddot{\varphi}_{2 k}=\varepsilon_{2 k}$ - instantaneous absolute angular accelerations of the dyad link (2).

By processing the mathematical model (2), the diagrams for the time variation of the linear displacements of the elastic rod were obtained in the Maple programming environment as shown by Fig. 4, for linear elastic longitudinal displacement, and by Fig. 5, for linear elastic transverse displacement.

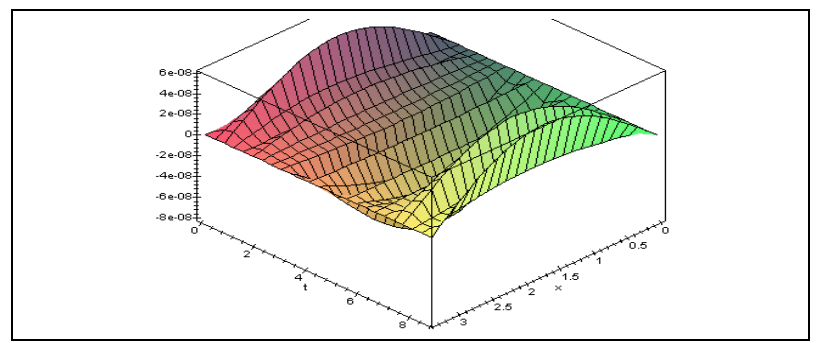

Fig. 4. Time variation diagram for longitudinal linear elastic displacements of link (2)

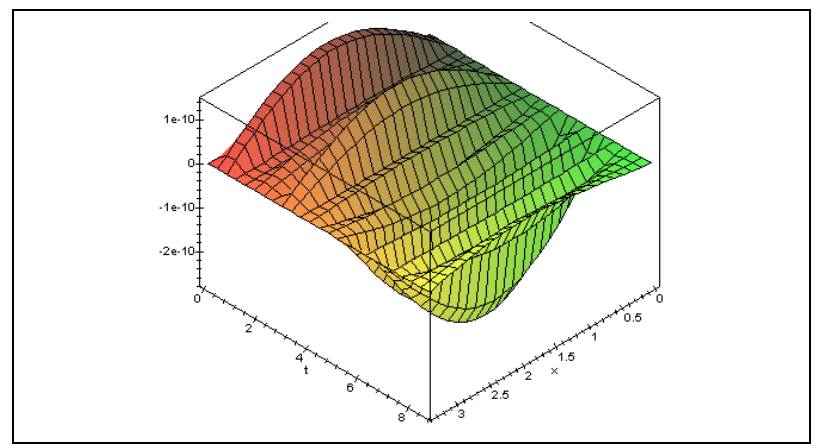

Fig. 5. Time variation diagram for transverse linear elastic displacements of link (2)
For processing the presented above mathematical models it is important the variation law of the angle of the pair $\mathrm{O}_{2}$ (curve 1), experimentally determined as shown in Fig. 6.

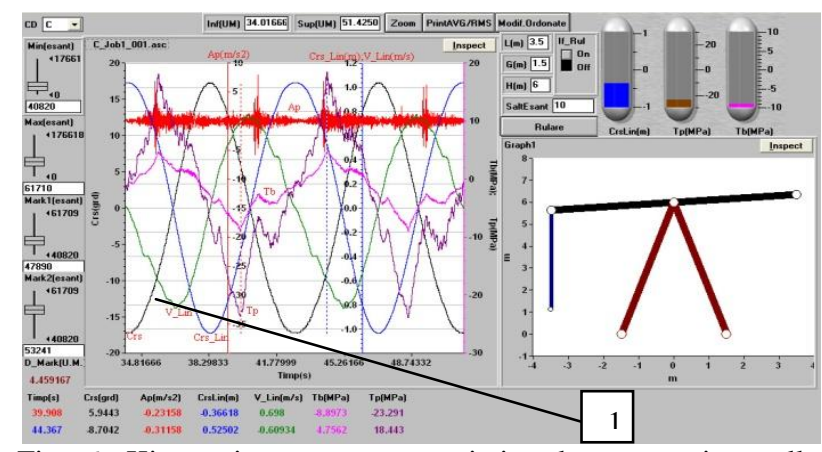

Fig. 6. Kinematic parameters variation laws experimentally determined

\section{CONCLUSION}

The purpose of this paper is a first version of the study, where the elastic constraint between the rigid element (3) and flexible element (4) was modeled by the revolute pair from the point $\mathrm{C}$.

There are still two options studied by the authors, namely, when the contact between the elements (3) and (4) is modeled by a pinion-rack type coupling and the real case, studying the contact between the rigid element (3) and the flexible element (4). This latest version will be analyzed using ADAMS software. Mathematical models for longitudinal and transverse vibrations of the mechanism two structural groups were developed and the variation laws diagrams of the vibration displacements were obtained. It was found that longitudinal and transverse elastic displacements are low; the obtained amplitude is justified by the high rigidity of the components of the mechanism first structural group.

Experimental research confirms this, but the important fact is that vibration data of a complex study of the pumping unit were obtained through this work. So, the time variation laws for kinematic parameters, respectively, positions, velocities and accelerations for the longitudinal and transverse vibrations were obtained. Based on the theoretical and experimental research presented above, the next step of the future research will regard the behavior analysis of the pumping system in thermalstructural coupled regime.

\section{REFERENCES}

Agirrebeita, J.; Fernandez-Bustos, I.; Ajuria, G. \& Angulo, C. (2006). A new finite element to represent prismatic joint constraints in mechanisms, SCIENCE DIRECT, Finite Elements in Analysis and Design, Volume 43, Issue 1, pp. 36-50, November 2006

Buculei, M. (1981). Mouvements aleatoires des mecanismes plans a elements deformables, Revue Roumaine des Sciences Techniques, Serie Mecanique Applique, 26, 325332

Dumitru, N.; Copilusi, C. \& Zuhair, A. (2009). Dynamic Modeling of a Mobile Mechanical System with Deformable Elements, Proceedings of the World Congress on Engineering, London, U.K, vol. II

Kuo, Y.L. \& Cleghorn, W.L. (2007). Application of Stressbased Finite Element Method to a Flexible Slider Crank Mechanism, 12 ${ }^{\text {th }}$ IFToMM Congress, Besancon, France, June 18-21, 2007

Liu, X.; Qi, Y.; Li, Y. \& Liu, C. (2011). An Approach to the Design Calculation of Sucker Rod Pumping Systems in Coalbed Methane Wells, Chinese Journal Of Mechanical Engineering, Vol. 24, DOI: 10.3901/ CJME.2011 